\title{
Pulse Shape effects on Backscattering Brillouin gain for distributed fiber sensing
}

\author{
C. Galíndez * J.M. Lázaro, P.B. García-Allende, F. Madruga, J.M. López-Higuera \\ Photonics Engineering Group, Universidad de Cantabria, Av. Castros s/n ETSII y de Teleco, \\ Santander 39005, SPAIN
}

\begin{abstract}
Distributed fiber sensing based on Brillouin gain scattering (BGS) principle is a useful way to develop devices capable to measure temperature and/or strain in optical fibers. In these distributed sensors, spatial resolution is a topic of special interest in the distributed fiber sensing field. The influence of the probe-pulse shape in the interaction between the pulsed light and the continuous wave laser in a pump-probe system. This study has the purpose of improving the spatial resolution of the measurement without losing stability in the BGS is presented. Also it is showed how the backscattering Brillouin gain is affected by inducing variations on the final value of the BGS intensity. Theoretical analysis of the probe pulse in the Brillouin shift and intensity values using triangular, sinusoidal and saw tooth shapes around the phonon lifetime ( 10ns) are presented; and also considerations and conclusions are explained.
\end{abstract}

Keywords: Brillouin gain scattering, pump pulse shape, pump probe interaction, continuous wave

\section{INTRODUCTION}

A proper pump light can cause fluctuations through electrostrictive mechanism in the core of an optical fiber, which induces the non linear Brillouin backscattering effect [1]. By using pulsed light, this effect is successfully employed to develop distributed sensing of temperature and/or strain along a fiber since the Brillouin frequency $v_{\mathrm{B}}$ is function of these two parameters. Both spontaneous or Stimulated Brillouin scattering can be used in the construction of sensors [2].

In a stimulated Brillouin scattering process a pulsed pump wave and a counter propagating probe wave interact. In distributed sensors the spatial resolution is one of the most important parameters. In stimulated brillouin sensors the spatial resolution can be determined by the interaction length between pulse and CW lights, which is half the pulse length. If the frequency of the CW is higher than that of the pulse, power is transferred from the CW to the pulse. If this light has a lower frequency, the power transfer is from the pulse to the CW light. The first configuration is referred to as the loss process since the CW light loses power, the second as the gain process because of the CW power gain [1, 3].

This work studies theoretically the interaction between the pulse and the probe light wave, where pulse shape is different to the typical square pulse. The interaction with sinc, triangular and saw tooth pulses are studied to analyse the accuracy of Brillouin measurements and the possible shape effects by using more realistic pulses generated in laboratory.

\section{THEORETICAL MODEL}

The description of the SBS process in optical fibers must consider mutual interaction between the pump and stokes waves, for a cw or quasi-cw pump. The steady state coupled-intensity equations for a slowly varying amplitude approximation of the interaction between the scattering and light fields can be expressed as follows [1, 3]:

$$
\frac{d I_{i}}{d z}=(\mp) g_{B} I_{i} I_{j} \pm \alpha I_{i} \text { with } i \neq j
$$

where the i-index ( 1 or 2 ) denotes the intensity of the pump or scattering light waves, $\alpha$ is the fiber attenuation coefficient and $\mathrm{g}_{\mathrm{B}}$ is the Brillouin gain factor. The sign for $\mathrm{g}_{\mathrm{B}}$ is negative for a Brillouin gain process and positive for $\mathrm{a}$

* galindezca@unican.es; phone 34942 200877; fax 34942200877

Third European Workshop on Optical Fibre Sensors, Antonello Cutolo, Brian Culshaw, José Miguel López-Higuera, Eds., Proceedings of SPIE Vol. 6619, 66193A, (2007) - 0277-786X/07/\$18 - doi: 10.1117/12.738669 
Brillouin loss process. The Brillouin spectral width is related to the damping time of acoustic waves or the phonon lifetime. In this case the acoustic waves are assumed to decay as $e^{-t / T_{B}}$, with $\mathrm{T}_{\mathrm{B}}$ as the phonon lifetime ( 10ns for optical fibers). BGS is described by a Lorentzian profile and the gain peaks at the Brillouin frequency $v_{\mathrm{B}}$ which is given by [1]

$$
g_{B}(v)=g_{0} \frac{(\Delta v / 2)^{2}}{\left(v-v_{B}\right)^{2}+(\Delta v / 2)^{2}} g_{0}=g_{B}\left(v_{B}\right)=\frac{2 \pi n^{7} p_{12}^{1} \gamma}{c \lambda^{2} \rho_{0} V_{a} \Delta v_{B}}
$$

where $\Delta v_{\mathrm{B}}$ is the full width at half maximum (FWHM) Brillouin line width and related to the phonon lifetime by $\Delta v_{B}=\left(\pi T_{B}\right)^{-1}, p_{12}$ is the longitudinal elasto-optic coefficient, $\rho_{0}$ is the material density, $\lambda$ is the pump light wave, $\mathrm{c}$ is the vacuum velocity of light. The frequency shift $v_{B}$ in the backward direction is given by $v_{B}=2 \pi V_{a} / \lambda$. The stokes intensity is found to grow exponentially in the backward direction according to $I_{2}(0)=I_{2}(L) e^{g_{0} P O L_{\text {eff }} / A_{\text {eff }}-\alpha L}$. This intensity can be express in terms of power by considering the power in the coupled equations 1 , thus the interaction can be write as

$$
P_{i}(z)=P_{i}(0) e^{\int_{L}^{x} a d z} e^{\int_{L}^{x}\left(-g_{B}(v, z)\right) P_{j} / A_{e f f} d z} \text { with } P_{i}=\int I_{i} d A
$$

where the integration area starts at the fiber length $\mathrm{L}$ and ends at the begining of the pulse wave, hence it is considered the region of the optical fiber where the pulse and pump waves interact; for the system in which both the gain and loss process occur, the total probe light power as a function of distance within the fiber can be expressed as $P_{1}=P_{1}^{+}(z)+P_{1}^{-}(z)$, where the first term on the right hand side is the power for the gain process and the second term is the power for the loss process and $A_{\text {eff }}$ is the effective fiber core area.
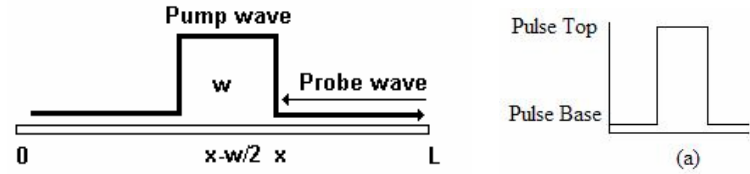

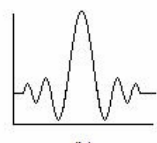

(b)

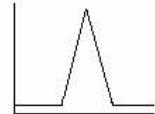

(c)

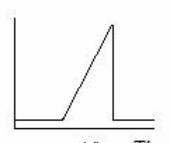

(d) Time

Fig. 1. Interaction between pump and probe light, (a) square pulse, (b) sinc pulse, (c) triangular pulse and (d) saw pulse

In order to use a single laser source the probe light wave is modulated at a fixed frequency using an external modulator, the modulation gives rise to new frequency lines in the optical spectrum (modulation sidebands). For pure intensity modulation, the obtained spectrum is symmetrical around the incident light wave frequency and the new lines are equally spaced in frequency. When the modulation frequency is equal to the Brillouin frequency shift $v_{\mathrm{B}}$, the first-order sidebands can interact with the incident light wave through SBS process, since they propagate in opposite directions. Pulses have maximum and minimum power different from zero, this situation is caused by the non perfect modulation, but if it considers that the pump light has no pulse base or it is neglected respect to the pulse top, the probe light interacts only with the highest part of the pulse, see Fig. 1.

\section{SIMULATION RESULTS AND DISCUSSION}

Simulations have been developed by using different pulses, which interact with the backscatter light, those pulses are: square, sinc, triangular, saw tooth and inverted saw tooth, see Fig. 1(a-d). The Brillouin gain factor (BGF) was simulated using equations 1 to 3 , the values used in simulation are: Refractive index $n=1.47$, Photo-elastic constant $p_{12}=0.22$ [3, 4], density $=2200 \mathrm{~kg} \mathrm{~m}^{-}$, acoustic wave velocity $V_{\mathrm{a}}=5550 \mathrm{~ms}^{-1}$, polarization factor $\gamma 0.68$ [4], attenuation coefficient of pump light $\alpha=0.046$, wavelength of the laser $\lambda=1553 \mathrm{~nm}$, effective fiber core area $A_{\mathrm{eff}}=50 \mu \mathrm{m}^{2}$.

BGF calculated numerically for each pulse shape is showed in fig 2. According to theory previously described, it is possible to note that the best response in Brillouin gain is for the square pulse, which presents an integration power area greater than the other shapes of similar pulse conditions of pulse top and pulse width power. But the pulse shape that really acts in an experimental set up looks more like a triangular, saw or sinc pulse; where the accuracy of the measurement is influenced both by the total interacting power and the spatial resolution. This situation depends on the pulse generator accuracy and its performance; thus it is convenient to simulate the variation of power and pulse width around phonon lifetime to analyze BGF using these pulse shapes. 

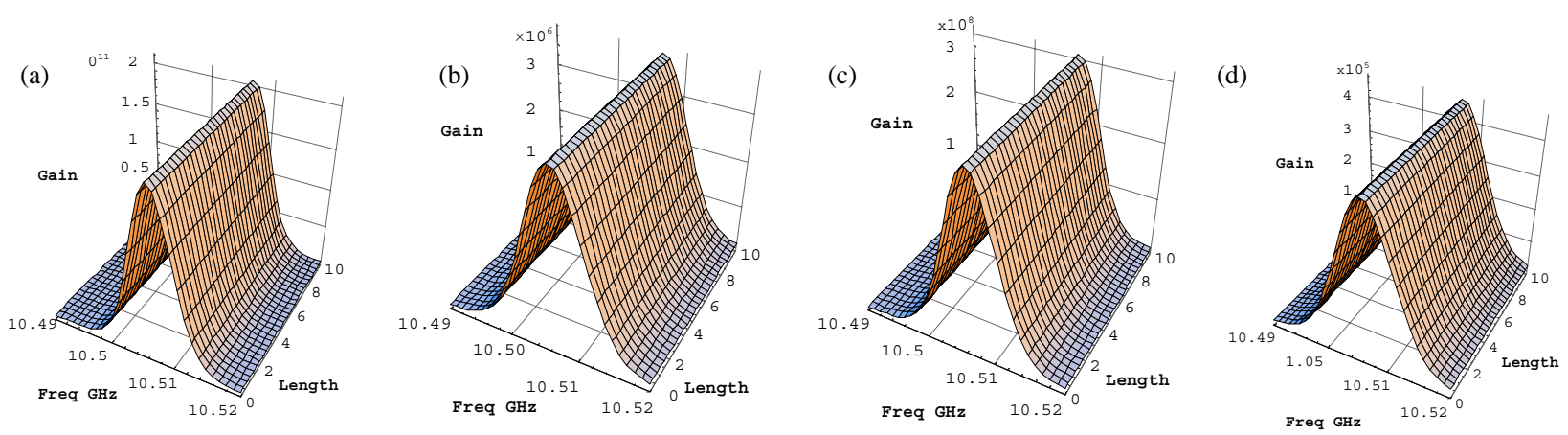

Fig. 2. Brillouin gain scattering obtained by (a) square, (b) sinc, (c) saw and (d) triangular pulses

Figure 3 shows the behavior of BGF when pulse shape varies in power and width. According to the numerical results obtained, if it is compared respect to square pulse, BGF has an exponential growth that depends on the distribution of the pulse power during the interaction process.

For a variation of pulse shape BGF presents a better response when the interaction is due to a saw pulse, the sinc pulse is better than the triangular, but quite far from the sinc pulse. This gain is enlarged and rapidly achieved when higher amounts of incident power are used. If the study is made for constant pulse width fixed at phonon lifetime of 10 ns and the power is changed around a constant value, the BGF response is similar to the pulse width analysis which presents an exponential increment that depends on the power and the Brillouin threshold. The best response is presented when the pulse has more power into the interaction area. Additional simulations for sinc and saw pulses are presented in Fig. 4.a to 4.d, in order to show the capability of using these pulses instead of the square.

In the graphics, it is important to note the presence of power and pulse width zones where BGF presents a better response; these zones could offer an increment on sensor sensitivity, as well as a better accuracy on the measurement of Brillouin frequency. This BGF response depends on an adequate combination of pulse shape, pulse power top and pulse width

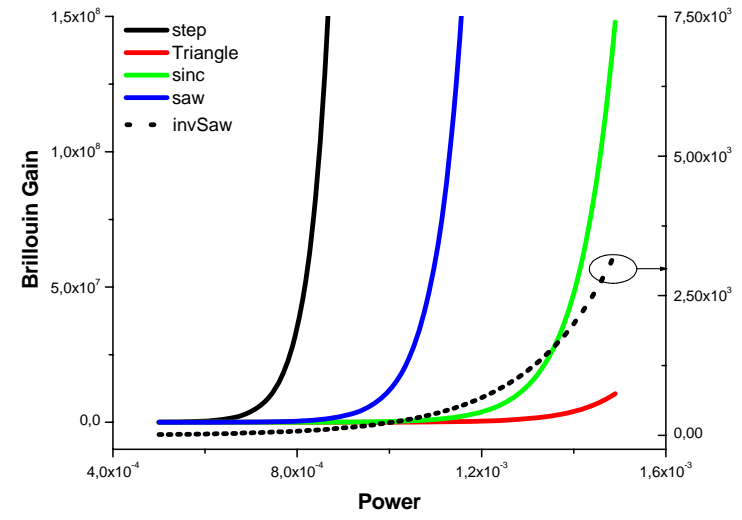

(a)

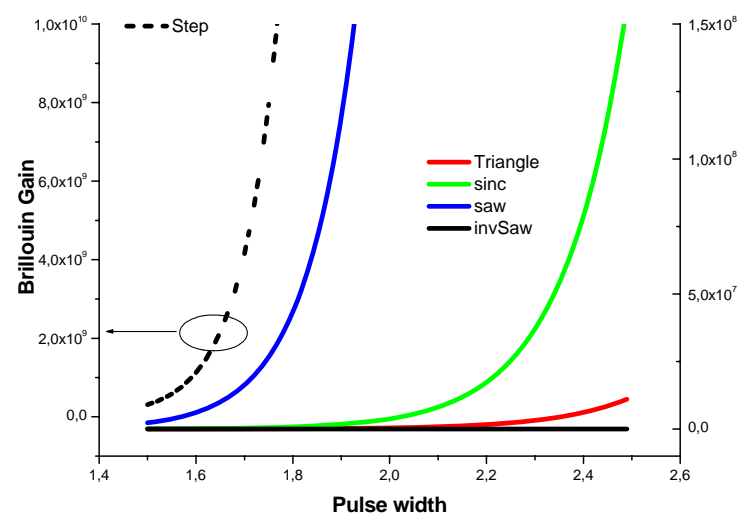

(b)

Fig. 3. Brillouin gain response. (a) power variation for 10ns pulse width and different pulses, (b) pulse width variation for pulses with power of $1.2 \mathrm{~mW}$.

\section{CONCLUSIONS}

The behavior of BGF when a pump field and different pulse shape fields interact in an optical fiber system was analyzed and studied theoretically in this paper. The results show that the interaction depends on the total amount of power involved during the nonlinear process. Since experimentally pulses are not perfectly squared, it is important to use pulses that present a better approximation, in order to improve the spatial resolution results. 
The results of simulation have been highly consistent with the experimental results reported in fiber sensing papers, which verifies properly the theoretical approach. The most important result of this simulation is that it is possible to use other pulse shapes like saw tooth to get similar results in Brillouin gain measurements. It only requires to meet a set of power and pulse conditions of saw tooth pulse close to its square pulse equivalent on power. An improvement on the spatial resolution could be achieved if the power is increased and the pulse width reduced for sinc or saw pulses.

Additionally, it is possible to use power zones where BGF presents a higher increment, if the sensor is placed around this work point; then, the sensitivity increases and the measurement could be more accurate.

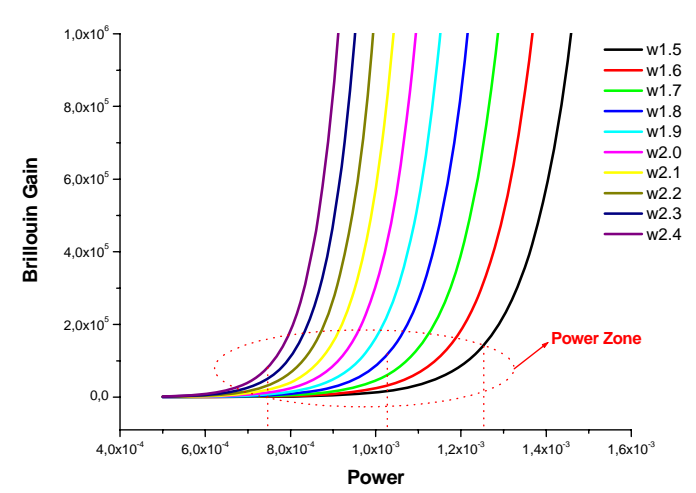

(a)

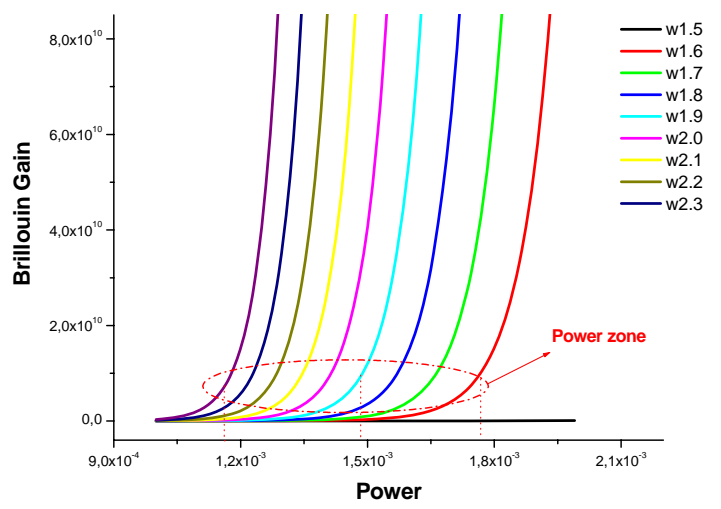

(c)

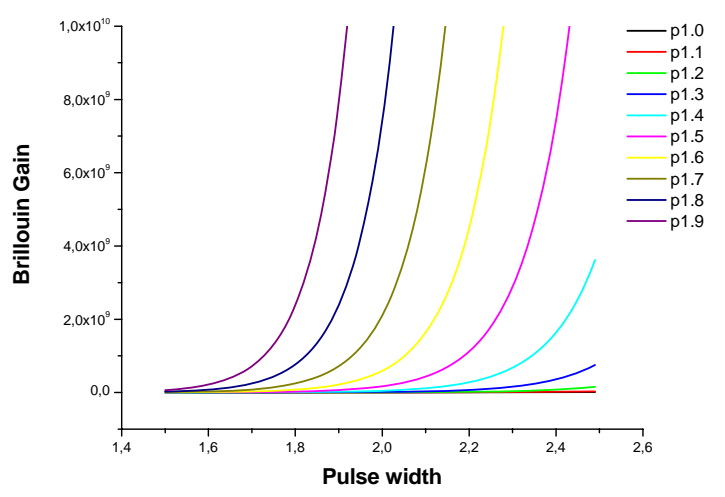

(b)

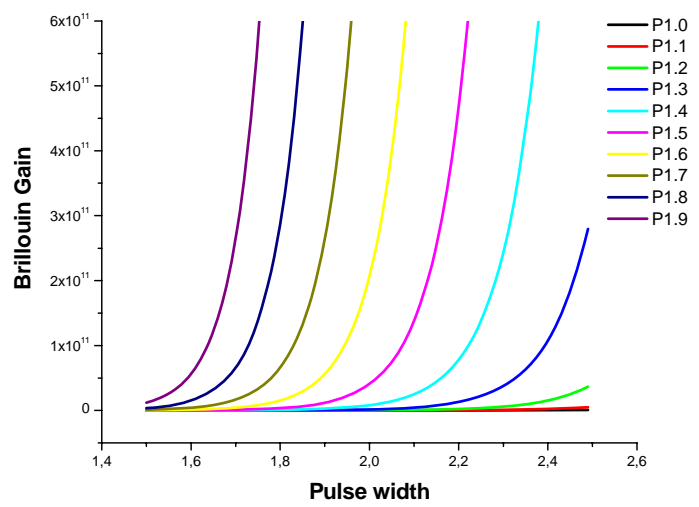

(d)

Fig. 4. Brillouin gain response. (a) variation of Power for sinc pulse at 10 widths, (b) variation of width for sinc pulse at 10 powers, (c) variation of Power for saw pulse at 10 widths, (d) variation of width for saw pulse at 10 powers.

\section{ACKNOWLEDGMENTS}

This work has been supported by the Spanish Government under the research project TEC2004-05936-CO2-02.

\section{REFERENCES}

1. $\quad$ Boyd, R.W., Non linear Optics. Second ed. 2003, USA: Academic Press; Elsevier Science.

2. Thévenaz, L. Review and progress in distributed fiber sensing. in OFS-18. 2006. Cancún, México: SPIE.

3. $\quad$ Agrawal, G.P., Nonlinear fiber optics. 1997, New York: Academic Press.

4. $\quad$ Cho, S.B., J.J. Lee, and I.B. Kwon, Pulse base effect on the strain measurement of a Brillouin-Scattering-based distributed optical fiber sensor. Smart Materials and Structures, 2006. 15: p. 10. 